\title{
Employees' Bottom Line Mentality and Safety Behaviors: The Moderating Role of Safety Climate
}

\author{
Shiwei Sun ${ }^{1, a}$, Han $\operatorname{Ren}^{2, b *}$ \\ ${ }^{1}$ Business School, Sichuan University, Chengdu, China \\ ${ }^{2}$ Business School, Sichuan University, Chengdu, China
}

\begin{abstract}
This paper is among the first to explore the relationship between employees' bottom line mentality (BLM) and safety behaviors, as well as the moderating role of safety climate in this relationship. Drawing on the social cognitive theory, we hypothesize that a) employees' BLM is positively correlated with safety behaviors; b) safety climate plays a significant moderating role between BLM and safety behaviors. We tested the data using AMOS and SPSS software, and the results from three-wave surveys of 275 employees provide broadly consistent support for our hypotheses. Theoretical and practical implications and future directions are also discussed.
\end{abstract}

\section{Introduction}

Bottom-line mentality (BLM) has been defined as "onedimensional thinking that revolves around securing bottom-line outcomes to the neglect of the competing priorities". Previous studies have focused on the negative effects of BLM. While its negative effects are certainly not negligible, these studies lack consideration for specific organizational situations.

For traditional production enterprises, "safety" is their bottom line. In this case, employees with BLM must more strictly abide by safety regulations in order to meet the performance targets. Therefore, this study focuses on the relationship of BLM and two dimensions of safety behaviors in traditional production enterprises. Meanwhile, we focus on the moderating effect of safety climate on the relation of BLM with safety behaviors. Specifically, we assume that employees' BLM will have a positive impact on safety behavior and safety climate moderates this relationship.

In order to test our hypotheses, we conducted three rounds of surveys, collected data from 275 Chinese employees, and tested hypotheses using hierarchical regression analysis.

\section{Theoretical Background and Hypotheses}

\subsection{Employee Bottom-Line Mentality and Safety Behavior}

Previous studies have focused on negative effects of BLM on leaders, employees and groups. By creating a BLM environment, supervisors may exacerbate workaholism and lead to cheating in the workplace [1]; for groups, the high BLM will lead to stronger adverse effects on group psychological security, thus reducing group creativity ${ }^{[2]}$. Obviously, previous studies focused on negative effects brought by BLM.

However, what remains unknown is whether BLM may result in positive outcomes in specific industrial contexts. In the traditional production and construction enterprises, employees' BLM may facilitate more safety behaviors. Drawing on the expectancy-valence theory, Neal posited that employees would have motivation to observe safety procedures and participate in safety activities if they believed that their actions would lead to worthwhile results ${ }^{[3]}$. Therefore, we propose that:

H1: Employees' BLM is positively related to safety behaviors.

Grinffin and Neal propose two subdimensions of safety behavior -- safety compliance and safety participation -- based on the distinction between task performance and situational performance ${ }^{[4]}$. Safety compliance refers to the core activities carried out by individuals to ensure the safety of the workplace, which can be embodied in compliance with standard working procedures, wearing personal protective equipment, etc ${ }^{[3]}$. Compared with other enterprises, traditional manufacturing enterprises face higher security risks. For example, the coal industry is the industry with the most safety accidents and the most serious casualties in China's industrial victory field ${ }^{[5]}$. For traditional production enterprises, employees with BLM can only complete the required outcome if they pay more attention to safety compliance.

On the other hand, safety participation is an extra-role behavior ${ }^{[6]}$ that does not directly contribute to personal safety. Instead, employees can effectively promote the development of a supportive environment through

b*Corresponding author. Email address: renhan@scu.edu.cn 
voluntary and active participation in safety activities, helping colleagues solve safety-related problems and participating in safety meetings ${ }^{[3]}$. Safety participation and safety compliance together constitute two dimensions of safety behavior. Similar to the foregoing discussion, safety participation is the basic requirement for employees to complete the outcome. Only employees who pay attention to safety participation and bottom-line can achieve the performance they value. Taken together, we propose the following hypotheses:

H2a: Employees' BLM is positively related to safety compliance

H2b: Employees' BLM is positively related to safety participation

\subsection{The moderating effect of Safety Climate}

Safety climate is a specific form of organizational climate that describes an individual's perception of the value of safety in the work environment (Neal, 2000). The five main factors of safety climate are management, safety system, risk, work pressure, and competence ${ }^{[7]}$. Brondino proposes that safety climate can be studied at both organizational and group levels ${ }^{[8]}$ : at the organizational level, safety atmosphere refers to employees' views on safety-related policies and procedures specified by top management; at the group level, the safety climate refers to the employee's perception of the practices of these policies and procedures that supervisors carry out in their daily work.

According to social cognitive theory, the relationship between attitude and behavior is influenced by situational factors. This paper argues that the leadership's advocacy and emphasis on safety constitute the contextual factor of the relationship between BLM and safety behavior. The specific manifestation of this contextual factor is as follows: when the leader attaches great importance to safety-related policies and procedures, employees' BLM can better promote the implementation of safety behavior; when leaders do not emphasize or pay attention to these policies and procedures of safety, employees' BLM may not facilitate their safe behaviors. To sum up, we propose the following hypotheses:

H3: Safety climate moderates the effect of employees' BLM on safety behavior.

H4a: Safety climate moderates the effect of employees' BLM on safety compliance.

H4b: Safety climate moderates the effect of employees' BLM on safety participation.

\section{Methods}

\subsection{Sample and procedures}

Employees from one large-scale state-owned coal enterprise located in North China participated in the study. To reduce the potential risk of common method bias, a three-time lagged design was employed ${ }^{[9]}$. At Time 1, a total of 320 employees reported their BLM and demographic variables. At Time 2 (one month later), questionnaires for measuring safety climate were distributed to the employees who completed the first survey and 292 valid data were collected. At Time 3 (after one month), 280 completed a questionnaire concerning the safety behavior, including safety compliance and safety participation. Eventually, 275 valid matched questionnaires were derived. Of the 275 employees, 91.1\% were male and $8.9 \%$ were female. Their average age was 39.05 , and they had an average of 15.26 years of organizational tenure. $33.3 \%$ of them had a university degree or above. $24.5 \%$ of the participants occupied managerial positions.

\subsection{Measures}

All key variables were rated using a 7-point Likert scale (from $1=$ strongly disagree to $7=$ strongly agree).

Employees' BLM. We used the BLM scale developed by Greenbaum (2010). The author modified the BLM items of employees to reflect employees' own BLM assessment rather than others' perception.

Safety climate. The safety climate was measured by the scale developed by Zohar ${ }^{[10]}$. We used 5 reverse coding items. One sample item of the scale is " As long as there is no accident, my supervisor doesn't care how the work is done ".

Safety behavior. Safety behavior was measured using a widely validated scale developed by Griffin ${ }^{[11]}$. This scale has two dimensions: safety compliance (4 items) and safety participation (3 items).

Control variables. We included gender, age, organizational tenure, education level, and position in the organization as control variables. Education level was measured as middle school or below, high school, college, university, and postgraduate. Organizational tenure was measured as the number of years worked in the current company. Position in the organization was measured using four categories: employees, first-line manager, middle manager, and senior manager.

\subsection{Results}

\section{Confirmatory factor analyses (CFA)}

A series of CFA procedures were conducted to evaluate the distinctiveness among the study variables through. Four variables were employed in our study: employees' BLM, safety climate, safety compliance, and safety participation. Using data obtained from the threewave surveys, we examined three alternative models against the baseline four-factor model (M1). As shown in Table 1, M1 fitted the data well $\left(\chi^{2} / d f=2.323\right.$, $\mathrm{SRMR}=.045, \quad \mathrm{RMSEA}=.069, \quad \mathrm{CFI}=.960, \quad \mathrm{IFI}=.961$, $\mathrm{NNFI}=.951)$ and provided substantial improvement in fit indices over alternative models (M2-4). 
Table1. Comparison of alternative measurement models

\begin{tabular}{|c|c|c|c|c|c|c|c|c|c|}
\hline Models & Factors & $\chi^{2}(d f)$ & $\chi^{2} / d f$ & $\triangle \chi^{2}$ & SRMR & RMSEA & CFI & IFI & NNFI \\
\hline 1 & Four factors: EBLM, SCli, SCom, SPar & $227.681(98)$ & $2.323 * *$ & -- & .045 & .069 & .960 & .961 & .951 \\
\hline 2 & $\begin{array}{l}\text { Three factors: SCom and SPar combined into one } \\
\text { factor }\end{array}$ & $430.648(101)$ & $4.264 * *$ & $202.967 * *$ & .074 & .109 & .899 & .900 & .880 \\
\hline 3 & $\begin{array}{l}\text { Two factors: EBLM and SCli, SCom and SPar } \\
\text { combined into one factor, respectively }\end{array}$ & $766.355(103)$ & $7.440 * *$ & $538.674 * *$ & .130 & .153 & .797 & .798 & .764 \\
\hline 4 & One factor: Four factors combined into one factor & $1889.408(324)$ & $18.167 * *$ & $1661.727 * *$ & .235 & .250 & .454 & .456 & .370 \\
\hline
\end{tabular}

Table2. Means, standard deviations, correlations, and scale reliabilities of all variables involved in the study

\begin{tabular}{|c|c|c|c|c|c|c|c|c|c|c|c|c|}
\hline Variables & Mean & SD & 1 & 2 & 3 & 4 & 5 & 6 & 7 & 8 & 9 & 10 \\
\hline 1 Gender & 1.09 & 0.29 & & & & & & & & & & \\
\hline 2 Age & 39.05 & 8.34 & 0.05 & & & & & & & & & \\
\hline 3 Tenure & 15.26 & 9.31 & $.142^{*}$ & $.859^{* *}$ & & & & & & & & \\
\hline 4 Education & 2.20 & 1.08 & $.232^{* *}$ & -0.021 & 0.089 & & & & & & & \\
\hline 5 Position & 1.32 & 0.61 & $.133^{*}$ & $.175^{* *}$ & $.222^{* *}$ & $.588^{* *}$ & & & & & & \\
\hline 6 Bottom Line Mentality & 3.80 & 1.28 & 0.001 & $.155^{*}$ & 0.102 & -0.079 & 0.081 & $(.820)$ & & & & \\
\hline 7 Group Safety Climate & 4.65 & 1.67 & 0.016 & -0.039 & 0.034 & $.166^{* *}$ & -0.033 & $-.275^{* *}$ & $(.953)$ & & & \\
\hline 8 Safety Ccompliance & 6.02 & 1.16 & $-.154^{*}$ & -0.021 & -0.086 & $-.239^{* *}$ & $-.184^{* *}$ & $.225^{* *}$ & $-.146^{*}$ & $(.935)$ & & \\
\hline 9 Safety Participation & 5.88 & 0.94 & -0.038 & 0.072 & 0.058 & -0.001 & $.125^{*}$ & 0.067 & -0.074 & $.450^{* *}$ & $(.779)$ & \\
\hline 10 Safety Behavior & 5.96 & 0.92 & $-.128^{*}$ & 0.017 & -0.037 & $-.173^{* *}$ & -0.078 & $.192^{* *}$ & $-.138^{*}$ & $.920^{* *}$ & $.764^{* *}$ & (.877) \\
\hline
\end{tabular}

\section{Hypotheses testing}

We used hierarchical regression analysis to test the hypotheses. Table 2 presents the means, standard deviations, reliabilities (Cronbach' alphas) and correlations of all variables. Table 3 shows the results of the hierarchical regression analysis. First, according to the results shown in the $M 2$, we found a significant relationship between employees' BLM and safety behavior
( $\beta=0.194, \mathrm{p}<0.01)$. These results provide support for $\mathrm{H} 1$. Secondly, M6 shows that the relationship between employees' BLM and safety compliance $(\beta=0.240, \mathrm{p}<0.01)$ was significantly positive, thus supporting $\mathrm{H} 2 \mathrm{a}$. However, in M10, we found that the relationship between employees' BLM and safety participation was not significant $(\beta=0.046$, n.s.), hence $\mathrm{H} 2 \mathrm{~b}$ was not supported.

Table3. Hierarchy regression results of all variables in the study

\begin{tabular}{|c|c|c|c|c|c|c|c|c|c|c|c|c|}
\hline \multirow{2}{*}{ Variables } & \multicolumn{4}{|c|}{ Safety behavior } & \multicolumn{4}{|c|}{ Safety compliance } & \multicolumn{4}{|c|}{ Safety participation } \\
\hline & M1 & M2 & M3 & M4 & M5 & M6 & M7 & M8 & M9 & M10 & M11 & M12 \\
\hline \multicolumn{13}{|l|}{ Control variables } \\
\hline 1 Gernder & -.074 & -.076 & -.077 & -.076 & -.080 & -.082 & -.084 & -.082 & -.037 & -.038 & -.038 & -.038 \\
\hline 2 Age & .158 & .138 & .135 & .165 & .161 & .136 & .133 & .167 & .095 & .090 & .089 & .100 \\
\hline 3 Tenure & -.177 & -.182 & -.176 & $-.207^{*}$ & $-.210^{*}$ & $-.217 *$ & $-.211 *$ & $-.246^{*}$ & -.056 & -.057 & -.054 & -.066 \\
\hline 4 Educational level & -.152 & -.118 & -.105 & -.072 & $-.156^{*}$ & -.114 & -.101 & -.063 & -.088 & -.080 & -.073 & -.060 \\
\hline 5 Position & .024 & -.007 & -.016 & .018 & -.074 & -.112 & -.122 & -.083 & $.177^{*}$ & $.170^{*}$ & $.164^{*}$ & $.177^{*}$ \\
\hline \multicolumn{13}{|l|}{ Independent variables } \\
\hline 6 Bottom line mentality & & $.194 * *$ & $.180 * *$ & $.190^{* *}$ & & $.240 * *$ & $.226 * *$ & $.237 * *$ & & .046 & .037 & .041 \\
\hline \multicolumn{13}{|l|}{ Mediator } \\
\hline 7 Group safety climate & & & -.056 & -.090 & & & -.058 & -.097 & & & -.033 & -.046 \\
\hline \multicolumn{13}{|l|}{ Moderator and interactor } \\
\hline 8 Bottom line mentality $\times$ group safety climate & & & & $.195 * *$ & & & & $.224 * *$ & & & & .074 \\
\hline Adjusted $R^{2}$ & .051 & .087 & .090 & .123 & .089 & .144 & .147 & .191 & .029 & .031 & .032 & .037 \\
\hline$\triangle \mathrm{R}^{2}$ & .051 & .036 & .003 & .033 & .089 & .055 & .003 & .044 & .029 & .002 & .001 & .005 \\
\hline$\triangle \mathrm{F}$ & $2.772^{* *}$ & $10.086^{* *}$ & $.780^{*}$ & $9.591^{* *}$ & $5.043^{* *}$ & $16.538^{* *}$ & $.883^{*}$ & $13.766^{* *}$ & $1.533^{*}$ & $.524^{*}$ & .248 & $1.272^{*}$ \\
\hline
\end{tabular}

To examine $\mathrm{H} 3,4 \mathrm{a}$ and $4 \mathrm{~b}$, we first calculated the centered values of employees' BLM and safety climate, and then constructed their interaction as BLM * safety climate. The results in Table 3 (see M4) show that the interaction term of BLM * safety climate exerts positive effect on employees' safety behavior $(\beta=0.195, \mathrm{p}<0.01)$, providing support for $\mathrm{H} 3$ Meanwhile, the relation between BLM * safety climate and safety compliance was significantly positive $(\beta=0.224, \mathrm{p}<0.01)$, which supported H4a. However, we did not find a significant relationship of BLM * safety climate with safety compliance $(\beta=0.074$, n.s.), thus H4b was not supported. 
To explicate this interaction, we drew separate plots for individuals whose scores were 1 SD below and above the mean of safety climate. Following Aiken and West's (1991) suggestion, Figure 1 and 2 shows the interaction effect between employees' BLM and safety climate on safety behavior and safety compliance. Particularly, the relationship between employees' BLM and safety behavior was positive and significant for the individuals with higher perceived safety climate $(\beta=0.336, p<0.01)$, and this relation was weakened and not significant for the individuals with lower perceived safety climate $(\beta=0.013$, n.s.). Similarly, the relationship between employees' BLM and safety compliance was positive and significant for the individuals with higher perceived safety climate $(\beta=0.440$, $\mathrm{p}<0.01)$, and this relation was weakened and not significant for the individuals with lower perceived safety climate $(\beta=0.034$, n.s. $)$.

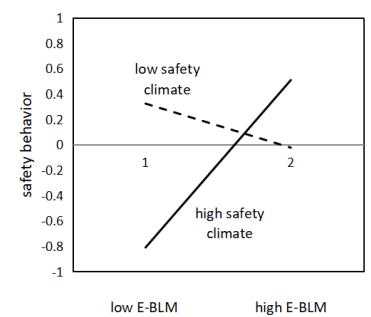

Fig 1. Interaction of employees' BLM (E-BLM) and perceived safety climate with safety behavior

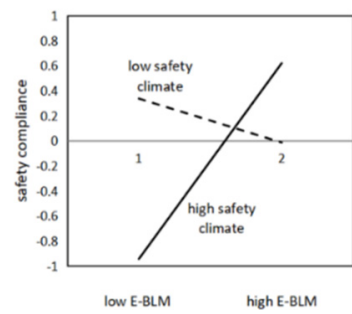

Fig 2. Interaction of employees' BLM (E-BLM) and perceived safety climate with safety compliance

\section{Discussion}

This study focused on the impact of employees' BLM on safety behaviors in traditional production enterprises, and the moderating role of safety climate. As hypothesized, we found that employee's BLM is positively correlated with safety behaviors and safety compliance, and the BLM of employees contributes to the safety behaviors and safety compliance. More importantly, the research results show that the safety climate moderate the relationship between employees' BLM and safety behaviors.

Theoretical contribution. Previous studies have mostly focused on negative effects of BLM, but lacked consideration of special circumstances in specific organizational situations. This research focuses on traditional production enterprises that attach great importance to safety, and examines positive effects of employees' BLM in traditional production enterprises on safety behavior and the moderating effect of safety climate. Specifically, this study proves the positive relationship between employees' BLM and safety behaviors in traditional production enterprises, and clarifies the moderating role of safety climate. Overall, our study enriches the research framework of BLM.

Practical implications. Our research demonstrated that in traditional manufacturing enterprise, employee's BLM has a positive impact on safety behavior. Therefore, the managers should pay attention to the BLM of employees. In addition, we found that safety climate moderates the relationship between employees' BLM and safety behaviors--when the safety climate is stronger, the positive effect of employees' BLM on safety behaviors is more obvious. Therefore, managers should pay attention to safety climate. It should be noted that safety climate is not created by employees. It should be the superiors who first set an example in safety to convey more convincing values to employees.

\section{Conclusion}

Our study explores the relationship between BLM and their safety behaviors, as well as the moderating role of safety climate. The results from three-wave surveys of 275 employees showed that BLM is positively correlated with safety behaviors, meanwhile safety climate can moderate this relationship such that the relationship between employees' BLM and safety behaviors is stronger when safety climate is higher. Our findings are of great importance to the management activities of safety production especially for those enterprises in the traditional industries.

Despite the theoretical contributions and practical implications of this research, it is essential to considered that employees are the only data source. Therefore, we acknowledge that the common source variance may be biased. We suggest that future research should include sufficient measurement time periods, and related variables should be measured from different perspectives such as employees and superiors to obtain more comprehensive result.

\section{References}

1. Farasat M, Azam A, Hassan H . Supervisor bottomline mentality, workaholism, and workplace cheating behavior: the moderating effect of employee entitlement. Ethics \& Behavior, 2020(4).

2. Greenbaum R L, Bonner J M, Mawritz M B, et al. It is all about the bottom line: Group bottom-line mentality, psychological safety, and group creativity. Journal of Organizational Behavior, 2020.

3. Andrew, Neal, Mark A, et al. A study of the lagged relationships among safety climate, safety motivation, safety behavior, and accidents at the individual and group levels.[J]. The Journal of applied psychology, 2006.

4. Griffin M A, Neal A . Perceptions of safety at work: a framework for linking safety climate to safety performance, knowledge, and motivation. Journal of Occupational Health Psychology, 2000, 5(3).

5. Chaoxian Guo. Production safety in Coal mine enterprises in China: based on the perspective of the quality of labor force. Chinese Industrial Economy 2007(10):105-112. (in Chinese)

6. Li, Yuanlong, et al. "Impact of Safety Attitude on the Safety Behavior of Coal Miners in China." Sustainability 11(2019). 
7. R. Flin et al. Measuring safety climate: identifying the common features[J]. Safety Science, 2000, 34(1) : 177-192.

8. Brondino M, Silva S A, Pasini M . Multilevel approach to organizational and group safety climate and safety performance: Co-workers as the missing link. Safety Science, 2012, 50(9).

9. Podsakoff, P. M., Mackenzie, S. B., Lee, J. Y., \& Podsakoff, N. P. (2003). Common method biases in behavioral research: A critical review of the literature and recommended remedies. Journal of Applied Psychology, 88(5), 879-903.

10. Zohar D . A group-level model of safety climate: testing the effect of group climate on microaccidents in manufacturing jobs. Journal of Applied Psychology, 2000, 85(4):587-96.

11. Griffin M A , Hu X . How leaders differentially motivate safety compliance and safety participation: The role of monitoring, inspiring, and learning. Safety Science, 2013, 60(Complete):196-202.

12. Aiken, L. S., \& West, S. G. (1991). Multiple regression: Testing and interpreting interactions. Evaluation Practice, 14(2), 167-168.

13. A Neal , M. A. G. B , and P. M. H. C . "The impact of organizational climate on safety climate and individual behavior." Safety Science 34. 13(2000):99-109.

14. Gabi E, Rebecca W, Scott W. L, et al. Winning at all costs: An exploration of bottom-line mentality, Machiavellianism, and organisational citizenship behaviour. Human Resource Management Journal, 2019, 29(3):469-489.

15. Greenbaum R L, Mawritz M B , Eissa G . Bottomline mentality as an antecedent of social undermining and the moderating roles of core self-evaluations and conscientiousness. Journal of Applied Psychology, 2012, 97(2):343.

16. Mirza M Z , Isha A S N . Safety-specific transformational leadership, safety climate and occupational accidents. 2020.

17. Quade M J , Mclarty B D , Bonner J M . The influence of supervisor bottom-line mentality and employee bottom-line mentality on leader-member exchange and subsequent employee performance. Human Relations, 2019(1):001872671985839. 